UDK 929Radics P.:94(436-89:497.4)

\title{
KULTURELLE SELBSTVERORTUNG UND IDENTITÄTSFRAGE: DER KRAINER POLYHISTOR PETER VON RADICS (1836-1912) ZWISCHEN ZWEI SPRACHEN UND KULTUREN
}

\author{
Tanja Žigon
}

\begin{abstract}
This article focuses on the life and creative path of the Carniolian polymath, many-faceted researcher, historian, German specialist, and theater specialist Peter von Radics (1836-1912), who was born in Postojna (Adelsberg) but did not have Carniolan roots. Based on this case study, the author examines the issues of what characterized an intellectual working in Carniola in the nineteenth century, what his place in society was, and what his understanding of his own identity was. The article also examines the hypothesis that one can speak of at least three identities in the nineteenth-century Slovenian lands - regional, ethnic, and dynastic (Habsburg), sheds light on the conditions that marked Radics' life and work, and seeks to explain why he chose to live in the microcosm of Carniola rather than seeking his fortune as a journalist, researcher, and author in the center of the monarchy, in Vienna.

Key words: Peter von Radics, Anastasius Grün, identity, cultural and literary history, Carniola, nineteenth century

Im Vorwort zum Drama aus dem 17. Jahrhundert Der verirrte Soldat, ${ }^{1}$ das Peter von Radics (1836-1912), ein vielseitig gebildeter und belesener Gelehrter und Theaterforscher aus Krain, während seiner Forschungen zum deutschen Theater in der Laiba-

\footnotetext{
${ }^{1}$ Die handschriftliche Fassung des Dramas, betitelt Der Verirrte Soldat oder Des Glück's Probierstein stammt aus der Feder der heimatlichen Verfasser Martin Höndler - nach Radics' Angaben soll er ein Gottscheer gewesen sein - und Melchior Harrer. Im Drama ist der Einfluss der Exotik auf die damalige Unterhaltungsliteratur zu erkennen, obwohl Peter von Radics in dem vorausgeschickten Vorwort diese Tatsache völlig außer Acht ließ. Die Handlung ist dem persischen Hofleben entnommen: Der von seinem eigenen Vater verworfene Prinz, kehrt nach sieben Jahren zurück, gerade in dem Augenblick, als sein Vater gegen den türkischen Sultan im Felde liegt, und rettet dem Vater das Leben. Zu guter Letzt verliebt sich der Prinz in die gefangene Sultanstochter, die er heimführt und heiratet. Das Drama wurde dem „Theatermäzen“, Vincenz Karl Fürst Auersperg (1812-1867) gewidmet. Besonders wertvoll in diesem Werk sind die angehängten Begleitstudien, die Peter von Radics selbst beisteuerte. Die erste Abhandlung bespricht die theatralischen Aufführungen in Ljubljana im XVII. und XVIII. Jahrhundert, die zweite ist ein Beitrag zur Geschichte der slowenischen Theatervorstellungen (Slovenische Dramen und die slovenischen Aufführungen im Laibacher Theater), von ihren Anfängen im Jahre 1789 bis 1865.
} 
cher Studienbibliothek aufstöberte und 1865 in Buchform herausgab (vgl. Žigon 2009: 257-262), konstatiert der Autor wie folgt:

Krain stellt, vom Standpunkte des Kulturhistorikers betrachtet, die Brücke vor, über welche romanische und deutsche Kunst von Nord nach Süd und in entgegen gesetzter Richtung die Jahrhunderte über gewandert sind, so kam es, dass die Wächter dieser Brücke, meine lieben slovenischen Landsleute, die eine wie die andere kennen lernten und in sich aufnahmen!

Vor allen andern Künsten war es aber ganz besonders die dramatische Kunst, die darüber hinzog, von den ,hochdeutschen Komödianten“ an, bis Shakespeare - Göthe - Schiller und von der wällischen Oper" bis Mozart!

Als das nationale Selbstbewusstsein zu Ende des vorigen Jahrhunderts auch bei uns in vieler erwachte, da geschah, wie natürlich, zugleich die erste Anregung: eine nationale dramatische Kunst zu schaffen! (Radics 1865: III).

Bereits der einleitende Satz spiegelt die kulturelle Realität im Land Krain im 19. Jahrhundert wider und bringt deutlich zum Ausdruck, dass Peter von Radics das Land Krain als eine Nahtstelle dreier Kulturen verstand. Er stellt fest, dass sich in Krain sowohl romanische als auch slawische und germanische Einflüsse verflechten, wobei vor allem die zwei letzterwähnten Kulturkreise Handel und Wandel in diesen Gebieten wesentlich geprägt haben. Ferner gewähren die zitierten Zeilen auch einen Einblick in die Selbstwahrnehmung und -verortung des Autors: Obwohl er seiner Abstammung nach kein Krainer war, nimmt er den Slowenen gegenüber nicht nur eine freundliche, wohlwollende Haltung ein, sondern er empfindet sich selbst als Krainer und voll integriert in der Krainer Gesellschaft, nennt die Slowenen seine „lieben Landsleute“ und drückt seine Zugehörigkeit mit dem empathischen ,bei uns“ aus.

Im vorliegenden Beitrag wird zunächst die kulturhistorische Situation im Land Krain skizziert. Anschließend werden anhand der Lebensgeschichte des berühmten Krainer Polyhistors Peter von Radics die Fragen beantwortet, was einen Krainer Intellektuellen im 19. Jahrhundert prägte und prädisponierte und wie sich seine kulturelle Selbstverortung und seine Identität definieren lassen, vor allem im Hinblick auf die Zerrissenheit zwischen dem Zentrum und der Peripherie: Einerseits stand nämlich den aus der Provinz stammenden Intellektuellen die Möglichkeit offen, sich in der Reichsund Hauptstadt Wien zu behaupten, andererseits waren ihre Erfolgschancen im Mikrokosmos der Provinz viel größer und realistischer. In diesem Sinne wird im Weiteren versucht festzustellen, wie soziale, ästhetische, ökonomische, mentale und kulturelle Interaktionen das Leben und Schaffen eines Einzelnen geprägt und bestimmt haben, ferner wird aber auch der Frage nachgegangen, wie sich ein Intellektueller aus den südlichen Gebieten des habsburgischen Vielvölkerstaates in der zweiten Hälfte des 19. und im angehenden 20. Jahrhundert zwischen Wien und Laibach verwirklicht und sich zwischen zwei Sprachen und Kulturen seinen Platz im kulturellen Leben der damaligen Zeit gesichert hat. 


\section{DER KULTURHISTORISCHE HINTERGRUND}

Nachdem die Habsburger im 14. und 15. Jahrhundert fast alle althabsburgischen Besitzungen in der Schweiz verloren hatten, erwarben sie 1335 nach dem Ableben des letzten Grafen von Tirol-Görz die Länder Kärnten und Krain (Hösler 2006: 33-38). Seit der zweiten Hälfte des 14. Jahrhunderts war Krain ein Herzogtum und von 1849 bis zum Ende des Ersten Weltkrieges ein österreichisches Kronland, das somit, ausgenommen die Zeit der Napoleonischen Illyrischen Provinzen (1809-1813), für fast 600 Jahre zu Österreich gehörte (vgl. ebd. 68-71).

Im Land Krain, wo die Mehrheit der Bevölkerung slowenischer Herkunft war, lebten Mitte des 19. Jahrhunderts 36 Prozent aller Slowenen, die anderen 64 Prozent bewohnten die angrenzenden Gebiete, die ebenfalls innerhalb der habsburgischen Hoheitsgrenzen lagen: die Untersteiermark, Kärnten, die Küstenregion, Görz, Gradisca und Istrien. Die Schwerpunkte der deutschsprachigen Besiedelungen waren vor allem die damaligen Landeshauptstädte, größere Städte in der Untersteiermark wie Marburg an der Drau (Maribor), Cilli (Celje), Pettau (Ptuj) (vgl. Brix 1988: 52) und die deutsche Sprachinsel Gottschee (Kočevsko) südöstlich von Ljubljana. Knapp zwei Drittel der Deutschen in Krain waren in der inselartigen Abgeschiedenheit des Gottscheerlandes (ebd. 55) angesiedelt, darüber hinaus lebten aber viele auch in der Hauptstadt Krains. Laut Statistik wohnten hier Mitte des 19. Jahrhunderts etwa 5.000 Deutsche, was ungefähr 40 Prozent der Gesamtbevölkerung in der Stadt ausmachte. Jedoch sank der Anteil der Deutschen in Krain von Zählung zu Zählung bis auf 27.915 Personen (5,4 Prozent) im Jahre 1910 (ebd. 54).

Die nationale Zugehörigkeit, Kultur und Sprache spielten im 19. Jahrhundert zunächst keine besondere Rolle im öffentlichen Diskurs. Die Phase einvernehmlicher deutsch-slowenischer Koexistenz und Symbiose dauerte bis in die 1880er Jahre hinein an. Erst zu dieser Zeit verschärfte sich die Reaktion der Deutschen in Krain. Es entstanden etliche deutschnationale Vereine, z. B. der Deutsche Schulverein (1880), der Deutsche Turnverein (1885) und die von deutschen Hochschullehrern gegründete Ferialverbindung „Carniola“ (1884). Jedoch lehnte das deutsche Lager in Krain bereits seit den 1860er Jahren und vor allem in der Zeit verstärkter nationaler und politischer Gegensätze eine pointiert deutschnationale Haltung ab. Stattdessen prononcierte man die liberale und verfassungstreue weltanschauliche Richtung (vgl. Vodopivec 1987: 31), kritisierte die slowenische nationale Partei als zu oppositionell, zu klerikal oder einfach als zu slawisch und protegierte die Vorrangstellung des Deutschen als Sprache mit langer kultureller Tradition.

Der slowenisch-deutsche Konflikt wurde Ende des 19. Jahrhunderts nicht nur auf dem politischen, sondern auch auf dem literarischen Felde ausgetragen. In diesem Zusammenhang kann die deutschsprachige Literatur in Krain als „Literatur der Grenze“ verstanden werden, im Unterschied zum früheren literarischen Konzept „Krain“, das die deutsche Kulturhegemonie mit einem paternalistischen Zugang zur slowenischen Literatur verband (Michler 1998: 204-213). Mit der politischen Konstellation hängt auch die nationale Vereinnahmung der Literatur zusammen. So wurde beispielsweise der oberösterreichische Lehrer an der Lehrer- und Lehrerinnenbildungsanstalt, Edward Samhaber (1846-1927), der 1878 nach Krain kam, nicht nur als großer Bewunderer 
des unbestritten bedeutendsten slowenischen Lyrikers aller Zeiten, France Prešeren (1800-1849), bekannt, sondern er verfasste in der Zeit seines zehnjährigen Aufenthalts in Ljubljana auch „Turnerlieder“ und Oden in denen er ,seine Zugehörigkeit zum Deutschtum hervorhob und seine Überzeugung von der Überlegenheit der deutschen Kultur gegenüber der slowenischen zum Ausdruck brachte“ (Miladinović Zalaznik 2000: 77).

$\mathrm{Zu}$ Beginn der 1880er Jahre zählte das damalige Ljubljana weniger als 30.000 Einwohner, wobei die Personen mit deutscher Umgangssprache 21,5 Prozent der Einwohnerschaft darstellten (Brix 1988: 55). Trotzdem hatte die Stadt nach außen hin eine deutsche Gestalt: Aufgrund der historischen Gegebenheiten wurde ungeachtet der Muttersprache und der Abstammung in der Oberschicht wie auch im bürgerlichen Milieu Deutsch gesprochen, die Straßenschilder waren überwiegend auf Deutsch, es wurden deutschsprachige Zeitungen und Zeitschriften herausgegeben und gelesen und auch den nationalbewussten Slowenen lag die deutsche Sprache noch immer näher als das noch nicht etablierte Slowenisch. Somit war die Umgangssprache in der Stadt Deutsch, worauf bereits im 17. Jahrhundert der Krainer Polyhistor Johann Weichart Freiherr von Valvasor (1641-1693), Mitglied der englischen Royal Society und Autor des monumentalen Werkes Die Ehre des Herzogthums Krain (1683), hinwies. Er stellte für Laibach fest, dass man hier untereinander sowohl Krainerisch als auch Deutsch spreche, ferner dass Adlige und Händler miteinander auch auf Italienisch kommunizierten, dass aber der schriftliche Verkehr in der Regel auf Deutsch geführt werde (Orožen 1993/94: 5). Das galt auch noch im 19. Jahrhundert, denn untereinander sprach man gewöhnlich deutsch, obwohl man auch slowenisch konnte.

In Anbetracht dieser Tatsachen spielte sich das literarische Leben in Krain entweder zwischen den oder in beiden Sprachen ab. So dichtete beispielsweise der bereits erwähnte France Prešeren, ,eine einsame dichterische Größe und ein anregender Denker der ersten Hälfte des 19. Jahrhunderts“ (Miladinović Zalaznik 1998: 91), nicht nur Slowenisch, sondern auch Deutsch, wobei seine deutschen Gedichte sogar von höherer literarischer Qualität sind als die von ihm ins Deutsche übertragenen eigenen slowenischen Lieder (ebd. 92). Dahingegen war sich die nach 1830 geborene Generation der slowenischen intellektuellen Elite, es seien hier nur die Schriftsteller Fran Levstik (1831-1887), Josip Jurčič (1844-1881) und Fran Erjavec (1834-1887) erwähnt, der bedeutenden Rolle der eigenen Muttersprache durchaus bewusst und bediente sich dieser so häufig wie möglich, jedoch kommunizierten ihre Vertreter sowohl mündlich als auch schriftlich untereinander überwiegend noch auf Deutsch. Das hängt wohl auch damit zusammen, dass slowenische Intellektuelle im 19. Jahrhundert gewöhnlich in Wien studierten; eine eigene slowenische Universität wurde erst im Jahre 1919, also nach dem Zerfall der Donaumonarchie, in Ljubljana gegründet.

Wien war für die aus den heutigen slowenischen Gebieten stammende Bevölkerung jahrhundertelang ein besonderer Anziehungspunkt. Hier versuchten viele Gelehrte, Wissensdurstige oder Handelsleute ihr Glück. Erstens erstarkte Wien bereits in der Zeit vom 11. bis zum 13. Jahrhundert zu einem blühenden und bedeutenden Handelszentrum (Zöllner 1990: 175-179), zweitens wurde im 14. Jahrhundert hier die zweitälteste Universität (nach der 1348 errichteten Karls-Universität in Prag) im deutschen Sprachraum gegründet. Danach kamen immer mehr Studenten aus den sloweni- 
schen Gebieten nach Wien. ${ }^{2}$ Im Vergleich zu der Prager Universität oder der italienischen Universität in Padova, die damals in Bezug auf die Entfernung genauso wie die Wiener Universität hätten in Frage kommen können, war das Studentenleben in Wien wesentlich billiger, weswegen auch Studenten aus ärmeren Schichten Zutritt zu der universitären Ausbildung hatten (Medved 1995: 23). Ferner trug aber auch gut überlegte Schulpolitik und später die durch Kaiserin Maria Theresia (1717-1780) verabschiedete Gesetzgebung dazu bei, dass die slowenische Intelligenz sich lieber für ein Studium an der Wiener als an anderen europäischen Universitäten entschied.

Bereits im 18. Jahrhundert trifft man in Wien auf viele bedeutende Persönlichkeiten aus den slowenischen Gebieten, wie z. B. auf den Naturwissenschaftler, Germanisten und in den Jahren 1753 und 1766 Professor der deutschen Sprache an der Wiener Universität, Žiga (Sigismund) Popovič (1705-1774) (vgl. Weiss 2007: 101-105), oder auf den berühmten Mathematiker und Astronomen, Jurij (Georg) Vega (1754-1802) (vgl. Pisanski 2006), den Verfasser des legendären Logaritmisch-trigonometrischen Handbuches (1780), das bis 1908 beachtliche 82 Auflagen erlebte. Ferner gehört zu den verdienstvollsten slowenischen Landsleuten, die in Wien Karriere machten und bedeutend das kulturelle Leben der Slowenen beeinflussten, auch der Sprachwissenschaftler Jernej (Bartholomäus) Kopitar (1780-1844) (vgl. Merchiers 2007; vgl. auch Grošelj 2008: 40-41), der zusammen mit Josef Dobrovský (1753-1829) (vgl. Grošelj 2008: 54) und Pavel Josef Šafárik (1795-1861) als einer der Begründer der wissenschaftlichen Slawistik gilt. Sein Zeitgenosse Fran Miklošič (dt. Franz Miklosich; 1813-1891) begründete 1849 die Wiener Slawistik und wurde fünf Jahre danach (1854/55) Rektor der Wiener Universität. Darüber hinaus studierten und lebten in Wien auch slowenische Klassiker: der Lyriker France Prešeren, der Schriftsteller und Literaturtheoretiker Josip Stritar (1836-1923) wie auch der größte Dichter der slowenischen Moderne, Ivan Cankar (1876-1918) (mehr zu diesem Thema Grdina 2013).

Aber nicht nur Wissenschaftler und Literaten verstanden die Residenzstadt des Vielvölkerstaates als Tor zur Welt, als ihr verheißenes Land. Die ökonomische Lage des 19. Jahrhunderts zwang auch viele Krainer dazu, sich vor allem in der Winterzeit nach Wien zu begeben, um dort überwiegend als Maronibrater (auf der Straße rösteten sie aber auch Kartoffeln und Äpfel) ihr Geld zu verdienen und sich so den Lebensunterhalt zu sichern. Die Wiener Jahre und Wien als eine ausgesprochen kosmopolitische Stadt, ein „Schmelztiegel“ verschiedener Kulturen und Religionen, haben jeden der Erwähnten nachhaltig geprägt und dadurch auf die Dauer auch das Leben in Krain weitgehend beeinflusst.

\section{PETER VON RADICS: BIOGRAPHISCHE TATSACHEN}

Peter von Radics wurde am 26. September 1836 in Adelsberg (Postojna) in Krain geboren und verstarb zwei Tage vor seinem 76. Geburtstag, am 24. September 1912 in Ljubljana. Seinem Geburtsort nach war Radics ein Krainer, seiner Abstammung

\footnotetext{
${ }^{2}$ Aus der ältesten Universitätsmatrikel geht hervor, dass in der Zeit zwischen 1377 bis 1400 an der Wiener Universität von ungefähr 600 Immatrikulierten 47 Studenten aus Laibach, 26 aus Cilli (Celje), 21 aus Pettau (Ptuj), 19 aus Krainburg (Kranj), 15 aus Marburg an der Drau (Maribor), 10 aus Gottschee (Kočevje) und 8 aus Stein (Kamnik) stammten (vgl. Medved 1995: 22; vgl. auch Cindrič 2009; Cindrič 2010).
} 
nach jedoch ein Deutscher. Er entstammte einer Mischehe. Seine Mutter, eine gewisse Leopoldine Binder (1796-1883) stammte aus Niederösterreich, sein Vater Peter von Radits (1779-1854) war aus Ungarn gebürtig, ,Sohn eines österreichischen Edelmannes", wie es in seinem Tagebuch, aufbewahrt im Slowenischen Nationalmuseum, steht (NMS, Nachlass Peter von Radics, TE 1 / II / C, Nr. 12). Er nahm bereits in den Kriegen gegen Napoleon auf der österreichischen Seite teil und ließ sich Ende der zwanziger Jahre des 19. Jahrhunderts in Adelsberg nieder, wo er als k. k. Straßenkommissar tätig war und für gute Verkehrsverbindungen und für die Aufsicht beim Straßenbau zuständig war (Žigon 2009: 28-30).

Darüber hinaus entstammte Peter von Radics einer Familie, die 1733 geadelt worden war und dem niederen Adel angehörte (ebd. 29). Trotzdem war sein Leben alles andere als adlig. Er musste hart um seine eigene Existenz und die seiner Familie kämpfen, sein tägliches Leben bestimmten finanzielle Schwierigkeiten und er musste sich deswegen mit Bienenfleiß der publizistischen Arbeit und dem Schreiben widmen.

Peter von Radics gehört zu den Autoren, die im 19. Jahrhundert in Krain wirkten, ihre Werke auf Deutsch verfassten und mit den bekanntesten Persönlichkeiten des politischen und literarischen Lebens der damaligen Zeit verkehrten. Er war ein in vielen Fächern bewanderter Gelehrter. Er war Publizist, Redakteur, Historiker, Germanist, Pädagoge, Ethnologe, Theaterforscher, mit einem Wort ein Polyhistor. Bereits zeit seines Lebens war er wegen seiner zahlreichen Studien zur Krainer Kultur-, Literatur-, Theater-, Sozial- wie auch Jagdgeschichte, Archiv-, Landes- und Erdkunde als der Krainer Historiograph bekannt. Er veröffentlichte mehr als 70 selbstständige Monographien und über 500 Zeitungsartikel und Beiträge zu den erwähnten Themen (vgl. ebd. 301-350). Auch wenn er keine bahnbrechenden Werke schuf, zählt er zweifelsohne zu den produktivsten Autoren seiner Zeit. Trotzdem schenkten ihm die lexikalischen Werke des 19. und angehenden 20. Jahrhunderts keine besondere Aufmerksamkeit. So hielt es beispielsweise sein Bekannter, der illustre Laibacher Bibliograph Constant von Wurzbach (1818-1893), nicht für notwendig, Radics in seinem monumentalen Lebenswerk, dem Bibliographische[n] Lexikon des Kaisertums Österreich (1856-1891) anzuführen, geschweige denn, ihm einen ganzen Lexikonartikel zu widmen. Genauso wird Radics auch in allen weiteren Lexika des 19. Jahrhunderts nur lapidar erwähnt (Hinrichsen 1891: 1074) und es werden nur knapp die wichtigsten bio- und bibliographischen Daten zu seiner Person zusammengestellt und veröffentlicht (Nagl et al. 1937: 1258).

Peter von Radics lebte und wirkte als Publizist sowohl in der Hauptstadt Krains als auch in Wien, er war der slowenischen Sprache mächtig, obwohl er seine Werke hauptsächlich auf Deutsch verfasste, er fühlte sich als Krainer, widmete sein gesamtes Opus seinem Geburtsland Krain, berief sich jedoch hier und da auch auf seine deutsche Abstammung und teilte mit dem Krainer Deutschtum Ende des 19. Jahrhunderts liberale Ideen, was ihm seine slowenischen Zeitgenossen verübelten. Sein Leben zwischen zwei Sprachen und Kulturen, wurde durch mehrere wichtige, jedoch ambivalente Faktoren gekennzeichnet, die ihn geprägt haben und im Weiteren beleuchtet werden. 


\section{DER REDAKTEUR IM DIENST DER VERSTÄNDIGUNG ZWISCHEN DEN KULTUREN}

Seine ersten Erfahrungen im Zeitungsgeschäft sammelte Peter von Radics in den sechziger Jahren des 19. Jahrhunderts in Ljubljana. Bereits vor 1865 wurde im slowenischen nationalen Lager die Gründung eines politischen Blattes diskutiert, wobei allem voran die Sprache des Blattes zur Diskussion stand. Letztendlich wurde seitens der Aktionäre, die die finanziellen Mittel zur Verfügung stellten, entschieden, ab Januar 1865 eine Zeitschrift in deutscher Sprache unter dem Titel Triglav. Zeitschrift für vaterländische Interessen herauszugeben. Die Redaktion übernahm im Einvernehmen mit den konservativen Altslowenen, vor allem nach der Absprache mit der führenden Persönlichkeit des damaligen slowenischen politischen Lebens, Johann Bleiweis (1808-1881), Peter von Radics (Žigon 2004: 41-116). In einer programmatischen Schrift, die auch in der ersten Nummer der Zeitschrift abgedruckt wurde, fasste er die Ziele des neuen publizistischen Organs kurz und bündig zusammen:

Den Deutschen [...], wie sie unter unserem Volke wohnen, dem die römischen Schriftsteller schon die Uebung der Gastfreundschaft zu hohem Lobe anrechneten, soll „Triglav“ für Besprechung berechtigter Interessen willig seine Spalten öffnen, denn wir wollen aufrichtig Verständigung und Versöhnung mit ihnen, deren Sprache wir uns, hauptsächlich im Hinblicke auf das ebengenannte schöne Ziel, in diesem unserm Organe bedienen (Radics 1865: 1).

Das Blatt, das sich laut seines Programms um ein besseres Verständnis zwischen dem Deutschtum und dem Slowenentum in Krain bemühte, geriet bald in Kritik und stieß vor allem bei der jüngeren Generation der Slowenen auf Widerstand. Peter von Radics, selbst überzeugt von der Notwendigkeit einer „vaterländischen“ Zeitschrift in deutscher Sprache, konnte sich unter diesen Umständen als Redakteur des unerwünschten Organs keine Sympathien erwerben. Nicht mal fünf Monate nach dem Erscheinen von Triglav, am 5. Mai 1865, bezog das Klagenfurter slowenische Blatt Slovenec [Der Slowene] Stellung zu der Laibacher Zeitschrift. Diese, so Slovenec, sei für das slowenische Volk vollkommen unnötig und schade dem slowenischen Volk mehr als es nütze (Malle 1979: 182). Die Angriffe wurden immer heftiger und bereits im Juni wurde auch Radics, der verantwortliche Redakteur des Triglavs, unter Beschuss genommen. Der „deutsche“ Triglav schäme sich seiner Muttersprache, konstatierte ein Korrespondent aus Krain und prophezeite dem Blatt ein baldiges Ende. Ferner hieß es, dass der Redakteur des Blattes „sein Mantel so zu drehen versteht, wie es seinem ,Dispositionsfonde' gefällt und der, Triglav' selbst sieht auch schon so aus, als ob er sich um einen Platz unter den Auserwählten des Wiener, Dispositionsfondes' bemühen würde" (ebd. 183).

Als im Jahre 1866 der Krieg zwischen Italien, Preußen und der Donaumonarchie ausbrach, traf Radics die Entscheidung, als Kriegsberichterstatter auf italienische Kriegsschauplätze zu ziehen. Im Frühling 1866 gab er die Redaktion auf und die Zeitschrift wurde vorübergehend eingestellt. Diesen Vorgang kommentierte das Klagenfurter Blatt Slovenec mit Blick auf die zuvor gemachte Prognose wie folgt: „Das 
slovenische Bewußtsein soll durch das slovenische Wort gekräftigt werden, das ist und bleibt unser Gedanke" (ebd.)

Ende der sechziger Jahre ging Radics nach Wien, enttäuscht und ernüchtert von seinen Erfahrungen in der Hauptstadt Krains, wo er feststellen musste, dass seine Ansichten, obwohl den Slowenen wohlgesinnt, nicht der Mehrheit der slowenischen Intelligenz der damaligen Zeit entsprachen. In Wien lernte er seine zukünftige Frau, Hedwig Kaltenbrunner (1845-1919), die Tochter des oberösterreichischen Mundartdichters Carl Adam Kaltenbrunner (1804-1867), kennen, heiratete und blieb zehn Jahre lang, von 1866 bis 1876, in der Haupt- und Residenzstadt. Er schrieb für diverse Wiener Zeitungen, war als Journalist und Redakteur tätig, jedoch konnte er sich in Wien nicht behaupten. Als im Jahre 1876 Gerüchte aufkamen, dass die Wiener Zeitung, der Österreichische Volksfreund, deren Mitarbeiter und zeitweise auch verantwortlicher Redakteur der Rubrik für Innenpolitik Radics war, sein Erscheinen einstellen würde, sah sich die Familie Radics genötigt, Wien zu verlassen und zurück nach Krain zu ziehen (Žigon 2009: 101-115). In dieser Hinsicht teilte Radics die Erfahrung vieler Intellektueller des 19. Jahrhunderts, die ihr Glück in der Hauptstadt der Donaumonarchie suchten, jedoch bald erkennen mussten, dass das Wirken in den jeweiligen Herkunftsländern manchmal die einzige Möglichkeit für ihr Überleben war. So kehrte auch Radics schließlich „,nach Hause“, nach Krain zurück und verbrachte hier die letzten 36 Jahre seines Lebens.

Radics selbst sah sich als einen „Krainer der alten Wurzeln“ (Anonym 1912: 4). Die emotional geladenen Verse des deutschen romantischen Lyrikers Johann Ludwig Wilhelm Müller (1794-1827), die er seinem Erstlingswerk über Adelsberg und seine Grotten vorausschickte, begleiteten ihn sein Leben lang und waren seine wichtigste Devise:

Es ist das kleinste Vaterland der größten Liebe nicht zu klein; Je enger es Dich rings umschließt, je näher wird's dem Herzen sein (Radics 1861: unpag.).

In diesem Sinne war seine Rückkehr aus Wien in das Geburtsland Krain, in seinen Mikrokosmos, gleichsam ein Weg nach Hause, ein Weg aus der Metropole in die heimische Provinz. Hier konnte Radics seine in Wien gesammelten publizistischen Erfahrungen gut einbringen. Er war zunächst im Zeitungsgeschäft tätig, widmete sich aber später der Erforschung der Krainer Geschichte und veröffentlichte einige bedeutende Texte, allen voran eine erste wissenschaftliche Monographie über den Gelehrten Johann Weichard Freiherr von Valvasor. Dieser Krainer Polyhistor aus dem 17. Jahrhundert stand nicht nur im Zentrum seines wissenschaftlichen Interesses, sondern Radics fühlte sich mit ihm auch persönlich verbunden, denn auch Valvasor beschäftigte sich zeit seines Lebens mit der slowenischen Vergangenheit, schrieb über Sitten und Bräuche der Slowenen, setzte sich mit den geographischen und topographischen Fragen auseinander und fühlte sich als Krainer, obwohl auch seine Eltern keine Krainer waren, sondern aus der Region Bergamo in Norditalien stammten. Bereits als junger Student begann Peter von Radics unermüdlich zu Valvasor zu recherchieren und er setzte seine Forschungen in den 1890er Jahren mit viel Eifer und Energie fort, um die noch fehlenden Quellen über den Polyhistor zu sammeln. Im Alter von 74 Jahren unternahm er noch eine Studienreise nach London, wo er im Archiv der Royal Society drei 
bis dahin unbekannte Briefe Valvasors eruierte und seine Monographie über Valvasor vollendete. Die Herausgabe finanzierte die Direktion des heimischen Geldinstituts, der Krainischen Sparkasse, was heutzutage in Slowenien die reinste Fiktion wäre. Mit seinem Lebenswerk, das zwei Jahre vor seinem Tod erschien, schuf Radics nicht nur eine breit gefächerte Grundlage für alle späteren wissenschaftlichen Auseinandersetzungen mit Valvasor, sondern es wurde ihm für sein Werk mit „Allerhöchster Entschließung“ vom 28. Februar 1910 auch der Titel eines Kaiserlichen Rates verliehen (mehr dazu Žigon 2009: 236- 237).

\section{GESCHEITERTE TRÄUME UND BEKANNTSCHAFT MIT ANASTASIUS GRÜN}

Ein entscheidender Wendepunkt im Leben von Peter von Radics kollidierte mit seiner festen Überzeugung, dass nur eine gute Ausbildung einem ein besseres und unbekümmertes Leben garantiert. Unglückliche Umstände ließen ihn diesbezüglich scheitern: Der zu frühe Tod seines Vaters, der daraus resultierende Mangel an finanziellen Mitteln und ständige Erkrankungen hinderten ihn daran, das in Wien angefangene Studium der Geschichte, Geographie und der deutschen Sprache abzuschließen und ein staatlich geprüfter Lehrer zu werden. Nachdem er sein Studium an den Nagel gehängt hatte, war es noch schwieriger für ihn, eine feste Arbeitstelle zu bekommen und mittellos, wie er war, konnte er auch unmöglich die Kosten für seine wissenschaftlichen Recherchen und Veröffentlichungen tragen. So wie viele seiner Zeitgenossen steckte er in einer finanziellen Klemme, aus der ihm ein guter Freund und großzügiger Mäzen, Anton Alexander Graf Auersperg alias Anastasius Grün (1807-1876), des Öfteren half (vgl. Žigon 2009).

Die Bekanntschaft mit Anastasius Grün (vgl. zu seiner Person Scharmitzer 2010), der mit seinem anonym erschienenen Lyrikzyklus Spaziergänge eines Wiener Poeten (Hamburg, 1831), Aufsehen erregte und in der Donaumonarchie Wellen schlug, ${ }^{3}$ hat das Leben von Peter von Radics entscheidend geprägt, zumal Grün an Radics und sein Talent glaubte und ihm in den düsteren Zeiten finanziell unter die Arme griff. Radics lernte Grün im Jahr 1857 in Graz im väterlichen Hause seines guten Freundes und Kommilitonen, des späteren Schriftstellers Leopold von Sacher-Masoch (1836-1895) kennen. Radics erinnert sich:

Es war an einem schönen, unvergesslichen Abende der Gesellschafts-Saison im Winter 1857/58, daß wir das Glück hatten, eben in dem Elternhause meines Freundes, in dem Salon des damaligen Polizeidirektors von Graz, des Herrn Hofrates Ritter von Sacher-Masoch, und zwar bei Gelegenheit einer daselbst von uns Studenten veranstalteten Theateraufführung, dem für alle schöngeistigen Bestrebungen Empfänglichen vorgestellt zu werden. Da war u. a. auch Holtei, der treffliche Shakespeare-Vorleser und zugleich

\footnotetext{
${ }^{3}$ Eine ausführliche Monographie zum Leben und Werk dieses Vormärzdichters ist anlässlich seines 200. Geburtstages auch in Ljubljana, seiner Geburtsstadt erschienen (Miladinović Zalaznik 2009; vgl. auch Miladinović Zalaznik 2012, 2013). Demnächst erscheint in der neuen Ausgabe des Slowenischen Biographischen Lexikons ein längerer Beitrag über Grün aus der Feder der gleichen Autorin.
} 
unser freiwilliger Dramaturg anwesend, der dann nach der, wie man sagte, vorzüglich gelungenen Aufführung von Goethes „Geschwistern“ - eines Lieblings-Repertoirestückes unserer kleinen Bühne - sich in das aus dem Munde Grün-Auerspergs gespendete Lob mit uns, wie letzterer scherzend hinzufügte, teilen konnte (Radics 1906: 93).

An einer anderen Stelle berichtet Radics, dass diese Begegnung auch bei Grün einen starken Eindruck hinterlassen hatte und in seinem Gedächtnis haften geblieben war. So bemerkte er bei einigen Gelegenheiten Radics gegenüber wie folgt: „Nicht wahr, es muß Sie eigentümlich berührt haben das Milieu, in dem Sie dem ,Wiener Spaziergänger' persönlich begegneten, im Hause - des Polizeidirektors!“‘ (ebd.)

In den sechziger Jahren, als Radics erfolglos eine feste Arbeitsstelle in Wien suchte, wandte er sich immer wieder an Anastasius Grün, der ihm als Mäzen so manches Mal aus einer prekären Lage geholfen hat. ${ }^{4}$ Allerdings machte sich Radics wegen der Bekanntschaft mit Grün keine Freunde im slowenischen Lager. Die Freundschaft mit dem liberalorientierten Auersperg, der beispielsweise 1863 im Krainer Landtag behauptete, dass Krain mit der ganzen ethnographischen Gruppe der Slowenen auf dem deutschen Kulturgebiet liege oder einige Jahre später, 1870, in einem Brief an den Krainer Politiker Karl Deschman (slow. Dragotin Dežman; 1821-1889) die Slowenen als „,moderne türkische Horden geistlich Be- und Verschnittener“" (Pivec-Stele 1930: 64) bezeichnete und die slowenische nationale Bewegung für ,pure Burleske“ (ebd. 66) hielt, sorgte eher dafür, dass Radics auch nach seinem Tode seine Verdienste im Bereich der slowenischen Kulturgeschichte abgesprochen wurden.

\section{DER ÖSTERREICHISCHE PATRIOTISMUS UND LIBERALE ANSICHTEN}

Ferner wurde das Leben und Werk von Peter von Radics grundlegend durch den österreichischen Patriotismus bestimmt. Am besten kann dies am folgenden Beispiel illustriert werden: Im Jahre 1874, zwei Jahre nach dem Tode von Franz Grillparzer, gehörte Radics zu den Mitbegründern des literarischen Grillparzer-Vereines (Žigon 2010). Die Aktivitäten des Vereines galten von der Person und dem Werk Grillparzers ausgehend, der Literatur-, Theater-, Kultur- und Geistesgeschichte der damaligen Zeit. Das wichtigste Ziel des Vereins war, wie Radics in einem Brief an die Schwestern Fröhlich schreibt, die ,hohe Verehrung für den unvergesslichen Dichter und Patrioten“ (WStLB, Brief v. 6. Januar 1874), der das Wesen der österreichischen Seele schlechthin verkörperte und einer ganzen menschlichen und kulturellen Stimmung persönlichen Ausdruck verlieh (Magris 2000: 119).

Für die slowenische Kultur- und Literaturgeschichte erscheint es von besonderer Bedeutung, dass die Gründer des Grillparzer-Vereins aus Krain kamen; neben Radics gehörte zum initiierenden Personenkreis auch noch der Slowene Ludwig Germonigg

\footnotetext{
${ }^{4}$ Aus Dankbarkeit hat Radics auch zwischen 1876 und 1906 zwei umfangreiche Arbeiten und mehr als ein Dutzend Zeitungsaufsätze über Grün verfasst. Als erstes ist 1876 in Stuttgart Radics' Festschrift zum siebzigsten Jubiläum des Dichters, Anastasius Grün und seine Heimath (Cottasche Buchhandlung in Stuttgart) erschienen. Dieser Arbeit folgte eine Monographie über Grüns Leben und Wirken, die zwei Jahre später, 1879, in Leipzig herausgegeben wurde. In allen Werken redet Radics über von Grün in den höchsten Tönen.
} 
(1823-1909). Die Gründer und die Mitglieder des Grillparzer-Vereines zeigten einen aufrichtigen habsburgischen Patriotismus und ihre Loyalität Österreich gegenüber. Darüber hinaus kann ihre Motivation auch als ein patriotischer Akt erklärt werden: Die Etablierung einer „österreichischen“ als „,nicht-reichsdeutschen“ Literatur unter der besonderen Berücksichtigung des Todes Grillparzers gerade zur Reichsgründung. In diesem Zusammenhang spielte auch die territoriale Identität „Krainer“ eine entscheidende Rolle. Die meisten, vor allem die gebildeten Slowenen im 19. Jahrhundert definierten sich zunächst als national-slowenisch und konkretisierten darauf ihre Zugehörigkeit als regionale bzw. Landeszugehörigkeit, d. h. sie waren z. B. Krainer oder Steirer. Allerdings ist bei den Slowenen auch eine überregionale, staatlich österreichische bzw. dynastisch habsburgische Verbundenheit festzustellen (Vodopivec 2001: 71-84). Sie waren österreichisch-ungarische Untertanen, die in ihrer imaginären Vorstellung den Kaiser als Vater der Nation idealisierten. Die Literatur der damaligen Zeit lebte und zehrte eben von der Erinnerung oder der Verteidigung eines „Mythos“, eben des Mythos von einer einstmals intakten, heilen, Schutz gewährenden Habsburger Kultur, wie das Claudio Magris (2000: 18) formuliert, und gerade dieser Mythos hatte auch für Radics und Germonik, die Gründer des Vereins, eine wichtige Bedeutung und einen besonderen Wert.

Als Letztes sind für das Leben und Wirken von Peter von Radics seine liberalen Ansichten von Bedeutung. Das Jahrhundert, im dem er lebte, wurde im europäischen Rahmen einerseits durch Kriege, andererseits durch die fortschreitende wirtschaftliche Entwicklung gekennzeichnet. Allmählich begeistert von dem Bau der Eisenbahnen, von der Modernisierung des Postwesens, der Einführung des Telegramms und des Telefons beschrieb er in seinen Artikeln die neuesten technischen Errungenschaften, was von seinem fortschrittlichen Denken zeugt. Auch im politischen Sinne identifizierte sich Radics mit den liberalen Forderungen: Er forderte persönliche, politische und religiöse Freiheit, eine Demokratisierung des Lebens auf allen Ebenen, die Einschränkung der Macht der Kirche und plädierte für Fortschritt und Modernisierung der Wirtschaft, was nicht immer im Sinne seiner eher konservativ orientierten slowenischen Freunde im Land Krain war.

\section{FAZIT}

Peter von Radics liebte sein Geburtsland Krain und es ist ihm zu verdanken, dass sowohl die historische Vergangenheit als auch die Kulturgeschichte Krains im ausgehenden 19. und im anbrechenden 20. Jahrhundert systematisch erforscht wurden. Er war in diesem Sinne das kulturelle und historische Gedächtnis des Landes Krain. Obwohl er seiner Abstammung nach kein Slowene war, fühlte er sich als Krainer und machte es sich zur Aufgabe, alles, was mit seinem Vaterland in Verbindung stand, aufzuschreiben, um es den kommenden Generationen zu erhalten. Bis heute sind seine Veröffentlichungen nicht in Vergessenheit geraten. Seine Werke verfasste er auf Deutsch und hat damit bewiesen, dass die Sprache allein nicht unbedingt ausschlaggebend für die nationale oder kulturelle Zugehörigkeit ist.

Peter von Radics versuchte sein Glück in Wien, er hat sich bemüht, seinen Raum unter der „Wiener Sonne“ zu finden, doch es wurde ihm dort kein Erfolg be- 
schert. Er teilte das Schicksal vieler Intellektueller seiner Zeit: In der Residenzstadt der Habsburger gab es kein Brot für ihn, er hatte zu wenige einflussreiche Beziehungen, zu viele Gegner und unzählige Konkurrenten, im kleinen Universum seines Vaterlandes Krain dagegen konnte er sich behaupten, überleben und bewundernswerte Erfolge erzielen und gehört zweifelsohne heute noch zu den produktivsten Autoren im 19. Jahrhundert in Krain.

Das Leben und Schaffen von Peter von Radics stellt einen Teil der slowenischen Vergangenheit dar. Er lebte in einem von Kämpfen um die Macht gezeichneten Zeitalter, in einer Zeit des unvorstellbaren wirtschaftlichen Fortschritts, aber auch in einer Zeit der sich zuspitzenden nationalen Verhältnisse in Österreich-Ungarn. Trotzdem hielt er an dem Konzept eines koexistenten Miteinanderlebens der verschiedenen Kulturen in der Donaumonarchie fest, weswegen er in Anbetracht der dominierenden politischen Anschauungen anachronistisch wirkte, seine Lebensdevise war bereits Ende des 19. Jahrhunderts überholt; sogar die „krainierische“ Identität, zu der er sich sein ganzes Leben lang bekannte, begann in den letzten Dekaden seines Lebens allmählich $\mathrm{zu}$ verschwinden.

Peter von Radics kann man abschließend als einen aufgeklärten Intellektuellen bezeichnen, der seine öffentliche Bestimmung darin sah, mehrere Kulturen miteinander zu verbinden, insbesondere die slowenische und die deutsche bzw. deutschösterreichische. Er verstand sich als Mittler und Vermittler zwischen den Kulturen, was ihm kurz vor seinem Tod mit der Verleihung des Titels Kaiserlicher Rat auch öffentlich anerkannt wurde und was ihm auch heute noch hoch angerechnet wird.

Universität Ljubljana, Slowenien

\section{QEULLEN- UND LITERATURVERZEICHNIS}

Anonym. „Peter Pavel pl. Radics umrl.“ Slovenec, Nr. 22, 25. September 1912, S. 4. Brix, Emil. „Die zahlenmäßige Präsenz des Deutschtums in den südslawischen Kronländern Cisleithaniens 1848-1918." Geschichte der Deutschen im Bereich des heutigen Slowenien 1848-1918. Hg. v. Helmut Rumpler und Arnold Suppan. Wien/ München: Verlag für Geschichte und Politik/R. Oldenbourg, 1988. 43-62.

Cindrič, Alojz. Študenti s Kranjske na dunajski univerzi 1848-1918. Ljubljana: Univerza v Ljubljani, 2009.

Cindrič, Alojz. Študenti s Kranjske na dunajski univerzi v prvi polovici 19. stoletja 1804-1848. Ljubljana: Univerza v Ljubljani, 2010.

Grdina, Igor. „Dunaj - domiva ali tujina? / Vienna - a native or foreign land?” Slovenski odnosi z Dunajem skozi čas. Hg. v. Vincenc Rajšp. Dunaj/Wien: Slovenski znanstveni institute/Slowenisches Wissenschaftsinstitut; Ljubljana: Založba ZRC, Inštitut za narodnostna vprašanja, 2013.

Grošelj, Robert. "Oblikoskladnja starocerkvenoslovanskega nepredložnega mestnika v luči razvoja slovničnega opisa." Jezikoslovni zapiski 14/2 (2008). 39-61.

Hinrichsen, Adolf. Das literarische Deutschland. Berlin: Verlag des „Literarischen Deutschlands“, 1891. 
Hösler, Joachim. Slowenien. Von den Anfängen bis zur Gegenwart. Regensburg/München: Verlag Friedrich Pustet; Südosteuropa-Gesellschaft, 2006.

Magris, Claudio. Der habsburgische Mythos in der modernen österreichischen Literatur. Wien: P. Zsolnay, 2000.

Malle, Auguštin. Die slowenische Presse in Kärnten 1848-1900. Wien: Slowenisches Wissenschaftsinstitut Wien, 1979.

Medved, Drago. Slovenski Dunaj. Celovec/Ljubljana/Dunaj: Mohorjeva založba, 1995.

Merchiers, Ingrid. Cultural nationalism in the South Slav Habsburg lands in the early nineteenth century. The scholarly network of Jernej Kopitar (1780-1844). München: Sagner, 2007.

Michler, Werner. „,Die Deutschen in Krain“. Literarisches Feld und nationaler Konflikt nach 1848." Zur Geschichte der österreichisch-slowenischen Literaturbeziehungen. Hg. v. Andreas Brandtner und Werner Michler. Wien: Turia+Kant, 1998. 193-219.

Miladinović Zalaznik, Mira. „Ein jedes Volk hat einen Mann, den es sich von einem heiligen reinen Glorienschein umgeben denkt'. Ein kleiner Versuch über France Prešeren im 19. Jahrhundert.“ Zur Geschichte der österreichisch-slowenischen Literaturbeziehungen. Hg. v. Andreas Brandtner und Werner Michler. Wien: Turia+Kant, 1998. 91-103.

Miladinović Zalaznik, Mira. „,Neben den Boxern in den illustrierten Zeitungen sehen die bekannten Schriftsteller schon miserabel genug aus: noch nicht Muskel und nicht mehr Geist.' Der österreichische Schriftsteller und Germanist Edward Samhaber (1846-1927) in Ljubljana." Aufbruch in die Moderne. Wechselbeziehungen und Kontroversen in der deutschsprachigen Literatur um die Jahrhundertwende im Donauraum. Hg. v. Anton Schwob und Zoltán Szendi. München: Südostdeutsches Kulturwerk 2000. 75-91.

Miladinović Zalaznik, Mira und Stane Granda (Hg.). Anton Aleksander grof Auersperg - Anastazij Grün. Razprave. Ljubljana: Nova revija, 2009.

Miladinović Zalaznik, Mira. „,Daß die großen Fragen [...] nicht ohne Mitwirkung der mächtigen Slawenfamilie nachhaltig zu lösen sind, hat [...] das weithin vernehmbare Rauschen der alten und vielästigen Slawenlinde deutlich genug angekündigt‘: Anton Alexander Graf Auersperg (1806-1876) - ein Gönner der Krainer Kulturszene." Gefühlswelten und Emotionsdiskurse in der deutschsprachigen Literatur. Hg. v. Kristian Donko und Neva Šlibar. Ljubljana: Znanstvena založba Filozofske fakultete, 2012. 57-69.

Miladinović Zalaznik, Mira: „Liberalec Anton Aleksander grof Turjaški - predmarčni pesnik Anastazij Grün in njegov odnos do Kranjcev." Heroji in slavne osebe na Slovenskem. Hg. v. Božidar Jezernik. Ljubljana: Znanstvena založba FF (Zupančičeva knjižnica 38). 65-88.

Nagl, Johann Williband, Jakob Zeidler und Eduard Castle (Hg.). Deutsch-österreichische Literaturgeschichte. Ein Handbuch zur Geschichte der deutschen Dichtung in Oesterreich-Ungarn. Bd. 4. Wien: Verlag von Carl Fromme, 1937.

NMS. Narodni muzej Slovenije [Slowenisches Nationalmuseum], Nachlass Peter von Radics, TE 1 / II / C, Nr. 12. 
Orožen, Martina. „Janez Vajkard Valvasor o slovenskem jeziku.“ Jezik in slovstvo 39 (1993/94). 3-12.

Pisanski, Tomaž et al. (Hg.). Jurij baron Vega in njegov čas. Zbornik ob 250-letnici rojstva/Baron Jurij Vega and His times: Celebrating 250 years. Ljubljana: DMFA, Arhiv Republike Slovenije, 2006.

Pivec-Stele, Melita. „Pisma Antona Auersperga Karlu Dežmanu.“ Glasnik Muzejskega društva za Slovenijo 11/1-4 (1930). 43-71.

Radics, Peter von. Adelsberg und seine Grotten. Triest: Lloyd, 1861.

Radics, Peter von. Der verirrte Soldat oder des Glück's Probierstein. Agram: Commissions-Verlag der Suppan'schen Buchhandlung, 1865.

Radics, Peter von. „Unser Programm.“ Triglav, Nr. 1, 3. Januar 1865, S. 1.

Radics, Peter von. „Erinnerungen an Anastasius Grün.“ Anastasius-Grün-Nummer der Laibacher Schulzeitung 34 (1906). 93-97.

Scharmitzer, Dietmar. Anastasius Grün (1806-1876). Leben und Werk. Wien/Köln/ Weimar: Böhlau, 2010.

Weiss, Peter. „Življenje in delo Janeza Žiga Valentina Popoviča.“ Glossarium Vindicum/ Janez Žiga Valentin Popovič (Johann Siegmund Valentin Popowitsch). Hg. v. Richrad Reutner und Peter Weiss. Ljubljana: Založba ZRC, 2007. 101-105.

Vodopivec, Peter. „O socialnih in gospodarskih nazorih nemškega meščanstva na Kranjskem od konca 60. do začetka 80. let 19. stoletja.“ Prispevki za novejšo zgodovino, XXVII/1-2 (1987). 7-32.

Vodopivec, Peter. „Slovenci v 19. stoletju: Miti in stvarnost.“ Temeljne prelomnice preteklih tisočletij. Hg. v. Stane Granda und Barbara Šatej. Ljubljana: Zveza zgodovinskih društev Slovenije, 2001.

WStLB. Wiener Stadt- und Landesbibliothek, Inv.-Nr. 73835: Brief von Peter von Radics an Ludwig August von Frankl-Hochwart vom 6. Januar 1874.

Zöllner, Erich. Geschichte Österreichs. Wien/München: Verlag für Geschichte und Politik/R. Oldenbourg, 1990.

Žigon, Tanja. Nemški časnik za slovenske interese - Triglav (1865-1870). Ljubljana: Zveza zgodovinskih društev Slovenije, 2004.

Žigon, Tanja. Zgodovinski spomin Kranjske. Življenje in delo Petra Pavla pl. Radicsa (1836-1912). Ljubljana: Zveza zgodovinskih društev Slovenije in Znanstvena založba FF, 2009.

Žigon, Tanja. „Anastazij Grün in Peter Pavel pl. Radics.” Anton Aleksander grof Auersperg - Anastazij Grün. Razprave. Hg. v. Mira Miladinović Zalaznik und Stane Granda. Ljubljana: Nova revija, 2009. 227-246.

Žigon, Tanja. „Der Grillparzer-Verein. Ein von Krainern gegründeter literarischer Zirkel in Wien." Literarische Zentrenbildung in Ostmittel- und Südosteuropa. Hg. v. Mira Miladinović Zalaznik, Maria Sass und Stefan Sienerth. München: IKGS 2010. 365-380. 